\title{
5-axis double-flank CNC machining of spiral bevel gears via custom-shaped milling tools - Part I: modeling and simulation
}

\author{
Pengbo Bo ${ }^{\mathrm{a}}$, Haizea González ${ }^{\mathrm{b}}$, Amaia Calleja ${ }^{\mathrm{b}}$, Luis Norberto López de Lacalle ${ }^{\mathrm{b}}$, Michael Bartoň ${ }^{*, \mathrm{c}}$ \\ ${ }^{a}$ School of Computer Science and Technology, Harbin Institute of Technology, \\ West Wenhua Street 2, 264209 Weihai, China \\ ${ }^{b}$ High Performance Manufacturing Group, Department of Mechanical Engineering, \\ the University of the Basque Country, Plaza Ingeniero Torres Quevedo 1, 48013, Bilbao, Basque Country, Spain \\ ${ }^{c} B C A M-$ Basque Center for Applied Mathematics, \\ Alameda de Mazarredo 14, 48009 Bilbao, Basque Country, Spain
}

\begin{abstract}
A new category of 5-axis flank computer numerically controlled (CNC) machining, called double-flank, is presented. Instead of using a predefined set of milling tools, we use the shape of the milling tool as a free parameter in our optimization-based approach and, for a given input free-form (NURBS) surface, compute a custom-shaped tool that admits highly-accurate machining. Aimed at curved narrow regions where the tool may have double tangential contact with the reference surface, like spiral bevel gears, the initial trajectory of the milling tool is estimated by fitting a ruled surface to the self-bisector of the reference surface. The shape of the tool and its motion then both undergo global optimization that seeks high approximation quality between the input free-form surface and its envelope approximation, fairness of the motion and the tool, and prevents overcutting. That is, our double-flank machining is meant for the semi-finishing stage and therefore the envelope of the motion is, by construction, penetration-free with the references surface. Our algorithm is validated by a commercial path-finding software and the prototype of the tool for a specific gear model is $3 \mathrm{D}$ printed.
\end{abstract}

Key words: 5-axis CNC machining, flank milling, custom-shaped tools, tangential movability, spiral bevel gears, free-form shape manufacturing

\section{Introduction \& Motivation}

Manufacturing of free-form surfaces is a substantial part in automobile and aircraft industries. Components like turbine blades, rotors, or gears are smooth, yet curvature-varying objects and their efficient manufacturing introduces several challenging problems in geometric modeling and computing because the very complex shape of these objects prevents them to be manufactured using simple straight cutting tools [1].

Computer numerically controlled (CNC) machining is the leading subtractive manufacturing technology, where the milling tool is navigated to move along a material block, removing the superfluous material, and forming the designed shape. In spite of blossoming technologies like 3D printing [2, 3], 5-axis CNC milling remains very important, especially for workpieces like turbine blisks or compressors [4] because these objects require high stiffness, and therefore manufacturing from a single material block is preferable when compared to the additive counterpart.

\footnotetext{
${ }^{*}$ Corresponding author

Email addresses: pbbo@hit.edu.cn (Pengbo Bo), haizea.gonzalez@ehu.eus (Haizea González), amaia.calleja@ehu.eus (Amaia Calleja), norberto. lzlacalle@ehu.eus (Luis Norberto López de Lacalle), mbarton@bcamath.org (Michael Bartoň)
}

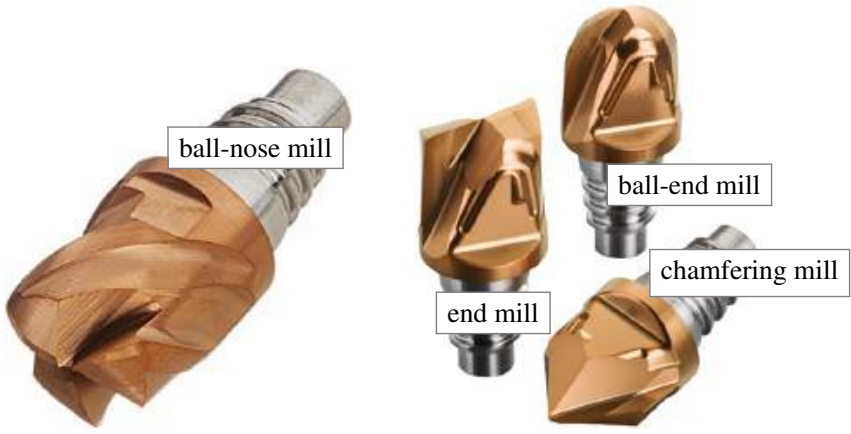

Figure 1: Tools' heads with various geometries. Figure courtesy of Sandvik Coromant.

CNC machining can be categorized depending (i) on the number of degrees freedom of the machine (e.g., 3-axis, 5axis), (ii) the type of the milling tool (flat-end, ball-end, bull nose, chamfering, general shape), see Fig. 1. or (iii) the type of contact between the tool and the material block (single point, multi-point, flank aka side or peripheral). Our study focuses on flank 5-axis machining with generally curved, custom-shaped milling tools.

Flank milling is typically the very finishing stage of the manufacturing process and high precision is indispensable. While for single-contact-point approaches the milling tool approximates the reference surface accurately only in the very neigh- 
borhood of the contact point, flank methodology aims at tangential contact between the tool and the surface. In such a case, the contact is theoretically attained along a 3D curve known as a grazing curve (or characteristic). This fact makes flank milling the dominant finishing technology because the milling tool matches the design shape at (theoretically infinitely) many points.

While traditionally the set of tools is given as an input, our approach looks not only for the optimal motion of the tool in 3D space, but also for the shape of the tool itself. We follow the approach introduced in [5] and look for a custom-shaped tool that, with its 3D motion, admits highly-accurate manufacturing.

\section{Previous work and contributions}

Tool path finding problem for 5-axis flank CNC machining with cylindrical milling tools can be alternatively formulated as approximating the offset surface of the input surface (offset by the cylinder's radius) by a set of ruled surfaces. Therefore a lot of literature is devoted to this equivalent formulation, see e.g. [6, 7, 8]. In general, representing a general free-form surface by a motion of a simple object as a cylinder or cone is theoretically not possible, unless the surface is an exact envelope. A decent simplification of the problem can be made by considering only ruled surfaces $[9,10,11,12,13,14,15,16]$ and using the argument that a free-form surface can be approximated by ruled surfaces arbitrarily well [6, 17]. However, this approximation of a general, doubly-curved surface by ruled surfaces within fine tolerances typically requires excessive number of patches [17].

It is therefore preferable to approximate a curved surface by an envelope of the milling tool directly, without inserting the simplifying step of approximating the surface by a ruled surface, followed by approximating the ruled surface by an envelope of a cone/cylinder [18]. One can describe an envelope of the milling tool using algebraic constraints and use an efficient subdivision solver [19]. Such an approach is computationally demanding, however, a full machining coverage as well as topological correctness is guaranteed by the solver.

Another important issue is the accessibility of the surface by a machining tool. A conservative estimate is proposed in the context of 5-axis ball-end milling [20]. The admissible directions of the tool are encoded using normal bounding cones which enables to quickly find whole volumes in the configuration space that correspond to possible tool paths. As a result, there is no need to compute accessibility for individual cutter contact points which brings significant computational savings.

In our previous research, we investigated the machinability of free-form surfaces using conical tools [21]. Using the initialization strategy for flank milling with conical tools introduced in [18], one quickly finds initial motions (ruled surfaces) of the milling axis and reveals the parts of free-form surfaces that can be efficiently approximated by conical envelopes within very fine machining tolerances. Consequently, high accuracy leads to a reduced machining time as only few sweeps are needed to cover large portions of the surface [21].

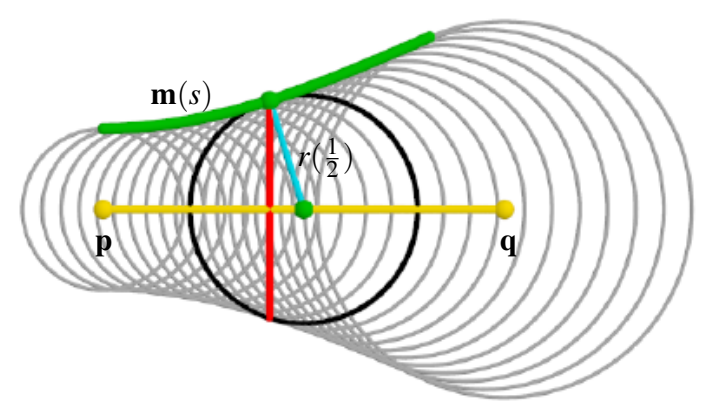

Figure 2: General meridian. A general surface of revolution is considered as a one-parameter family of spheres; a situation in an axial plane (2D view). The spheres are centered along the axis $\mathbf{l}=\mathbf{p q}$ and the meridian (green) touches one-parameter family of circles. The surface of revolution $\Psi$ arises by rotating $\mathbf{m}$ around $\mathbf{l}$. The sphere centered at the midpoint of $\mathbf{l}$ (black) touches $\Psi$ along a circle that projects as a straight line (red).

Another research category closely related to our research deals with barrel tools [1, 22, 23]. Barrel tools are shown to fit well free-form surfaces, especially in concave regions where the principal curvatures of the tool match their counterparts of the surface. However, in contrast to our work, the shape of the tool is given as the input while our approach seeks not only the 3D motion but the shape of the tool as well.

Another relevant class of research deals with inspection stage of an already-machined free-form surface. Typically mechanical and optical inspection machines are used; our research is related to 5-axis, contact based (mechanical) inspection path planning. Various path planning algorithms exist, we refer the reader to [24] and the references cited therein.

Optimizing simultaneously both the tool trajectory and its size and/or shape is a very active research area [25, 26, 27, 28, 29, 30] and our research belongs to this category of algorithms. To the best of our knowledge, all previous research requires the initial milling trajectory as a part of the input or is indicated by the user. Our recent research focuses on automatic path initiliazation for 5-axis flank milling [18, 31]. For a specific shape of the milling tool (conical or curved), an automatic initialization of the tool's motion can be achieved by integrating the admissible multi-valued vector field that corresponds to directions in which the point-surface distance changes according to the prescribed shape of the milling tool (prescribed by a meridian curve) [31]. As in this work the tool path is very much constrained by the reference geometry, we look for initial path that closely approximate the surface self-bisector. To this end, the most convenient approach is to consider a general surface of revolution as a one-parameter family of spheres [32], see Fig 2 .

In this paper, we introduce a new category of 5-axis CNCmachining, namely double-flank milling using custom-shaped milling tools. This methodology can be seen as an alternative to face hobbing where two sets of blades are needed to carve the space between two teeth of a bevel gear. In contrast, doubleflank machining is aimed for machining of curved narrow valleys, like e.g. gears with curved teeth, using a single customshaped tool. Such a machining strategy is more efficient than traditional (one-sided) flank milling as it removes material on 
two sides of the reference geometry by a single sweep of the tool. This methodology is aimed for semi-finishing stage, where high accuracy and, at the same time, high material removal rates are required. In our optimization-based approach, we design a custom-shaped tool and its motion such that it maximizes material removal, yet is by construction penetration-free (i.e., avoids overcutting).

The rest of the paper is organized as follows. Section 3 gives a mathematical description of rigid body kinematics of surfaces of revolution and Section 4 presents the algorithm that computes the envelopes of these surfaces to approximate the input free-form geometry. Section 5 shows our computer simulations, followed by validations using a commercial path-finding stateof-the-art software Siemens NX, and 3D printed prototype of a custom-shaped milling tool. Finally, Section 6 discusses the future research directions and concludes the paper.

\section{Basics of kinematic geometry}

We first recall several facts from kinematic and differential geometry, namely surfaces of revolution, the first order analysis of rigid body motions, and envelopes of surfaces of revolution.

\subsection{Surfaces of revolution}

Let $\mathbf{l}:=\mathbf{p q}$ be a finite 3D line and let $\mathbf{m}$ be a planar curve that lies in a plane containing $\mathbf{l}$. Rotating $\mathbf{m}$ around $\mathbf{l}$ gives rise to a surface of revolution $\Psi$. Line $\mathbf{l}$ is known as the axis of $\Psi$ and $\mathbf{m}$ as its half-meridian, see Fig. 2 An alternative point of view is to consider a one-parameter family of spheres centered along $\mathbf{l}$. Then $\Psi$ is the envelope of the family of spheres and, if the sphere radius $r(s)$ is "well-behaved" (see Remark 1), each sphere touches $\Psi$ along a circle that lies in a plane perpendicular to $\mathbf{l}$.

Remark 1. From practical point of view, one needs to exclude pathological cases, e.g. when the one parameter family of spheres is locally contained by another sphere. By "well-behaved" we understand that the sphere radius $r$ is is not expanding/shrinking too quickly when considered as a function of the arc length $s$ of the axis, i.e., $\mid\left(r^{\prime}(s) \mid<1\right.$.

\subsection{Kinematics of a rigid body}

To control the motion of the milling tool $\Psi$, it is sufficient to control the motion of its rigid axis pq. Let us consider pq as a function of time and let us denote $\mathbf{p}(t)$ and $\mathbf{q}(t)$ the trajectories of the endpoints, $t \in[0,1]$. Since 1 remains rigid during the motion, the distance-preserving constraint of its length $L=\|\mathbf{p}(t)-\mathbf{q}(t)\|$ reads as

$$
\|\mathbf{p}-\mathbf{q}\|^{2}=\langle\mathbf{p}-\mathbf{q}, \mathbf{p}-\mathbf{q}\rangle=\text { const. }
$$

where $\langle\cdot, \cdot\rangle$ is the scalar product. Differentiating with respect to $t$ and denoting the velocity vectors by $\mathbf{v}_{\mathbf{p}}=\dot{\mathbf{p}}(t), \mathbf{v}_{\mathbf{q}}=\dot{\mathbf{q}}(t)$, the first order length-preserving constraint of $\mathbf{l}$ becomes

$$
\left\langle\mathbf{v}_{\mathbf{p}}, \mathbf{p}-\mathbf{q}\right\rangle=\left\langle\mathbf{v}_{\mathbf{q}}, \mathbf{p}-\mathbf{q}\right\rangle
$$

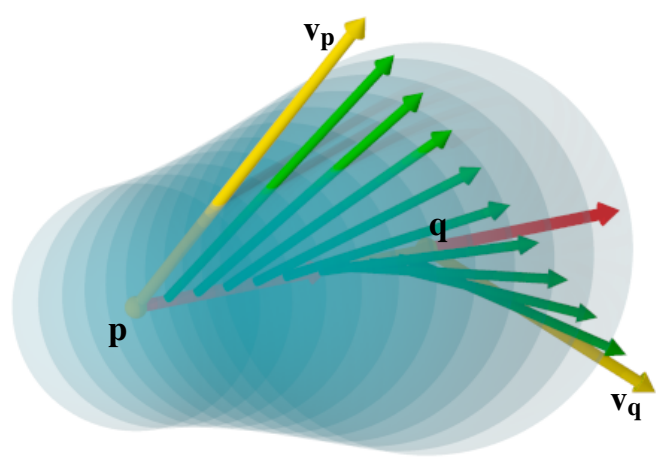

Figure 3: A generally-curved surface of revolution $\Psi$ is considered as an envelope of a one-parameter family of spheres (transparent) centered on the axis pq. An instantaneous motion of the rigid axis is determined by two velocity vectors (yellow) that satisfy Eq. 2, i.e., their projections onto pq are equally oriented vectors of the same length (red).

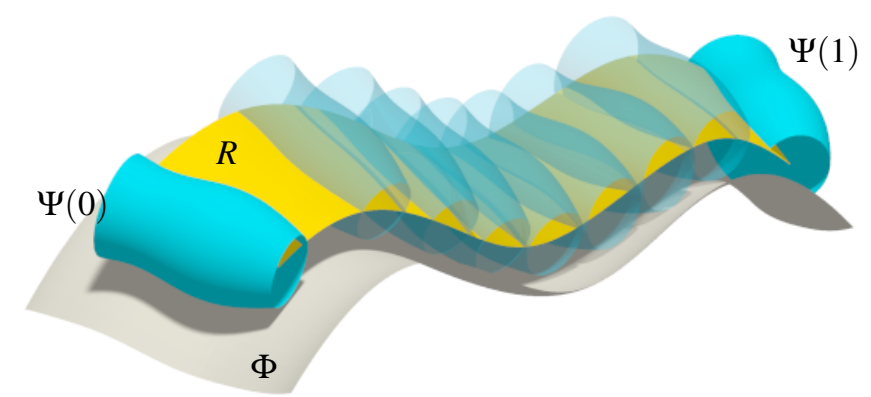

Figure 4: Given a free-form surface $\Phi$, the goal of (one-sided) flank CNC machining aims to find a generally curved surface of revolution $\Psi$ and its motion such that the envelope approximates $\Phi$ within a machining tolerance. The motion of the axis of the tool is visualized as a ruled surface, $R$, (yellow).

which is known as a projection rule, see Fig 3 .

The instantaneous motion of $\mathbf{l}$ can be conveniently controlled by a pair of vectors $\mathbf{v}_{\mathbf{p}}, \mathbf{v}_{\mathbf{q}}$ that satisfy Eq. 2, see Fig. 3 . The vector field that acts on the points of $\mathbf{l}$ is linear in $\mathbf{v}_{\mathbf{p}}$ and $\mathbf{v}_{\mathbf{q}}$ which follows from the fact that the instantaneous motion (aka screw) can be expressed as

$$
\mathbf{v}_{\mathbf{z}}=\overline{\mathbf{c}}+\mathbf{c} \times \mathbf{z}
$$

where $\mathbf{z}$ is a point in $3 \mathrm{D}, \mathbf{v}_{\mathbf{z}}$ its velocity vector, and $\overline{\mathbf{c}}, \mathbf{c} \in \mathbb{R}^{3}$, are constant vectors that define the instantaneous motion (the screw axis and the pitch), see e.g. [33] for more details.

\subsection{Envelopes of surfaces of revolution}

Consider now a motion of $\Psi$ in $3 \mathrm{D}$ space, that is, $\Psi$ is a function of time $t$, and without loss of generality assume that $t \in[0,1]$. The motion of $\Psi$ is governed by the axis $\mathbf{I}$ and its trajectory generates a ruled surface $R$, see Fig. 4 . A surface that touches the one-parameter family of $\Psi \mathrm{s}$ is known as the envelope, $\Omega$, which typically consists of two parts, the upper and lower patch. Given a free-form surface $\Phi$, our goal is to approximate it by the envelope within fine machining tolerances.

Remark 2. The envelope $\Omega$ need not to consist of two branches. As an counter-example consider a translation of $\Psi$ in the direction of $\mathbf{l}$. The envelope is a cylinder then, but we disregard these 


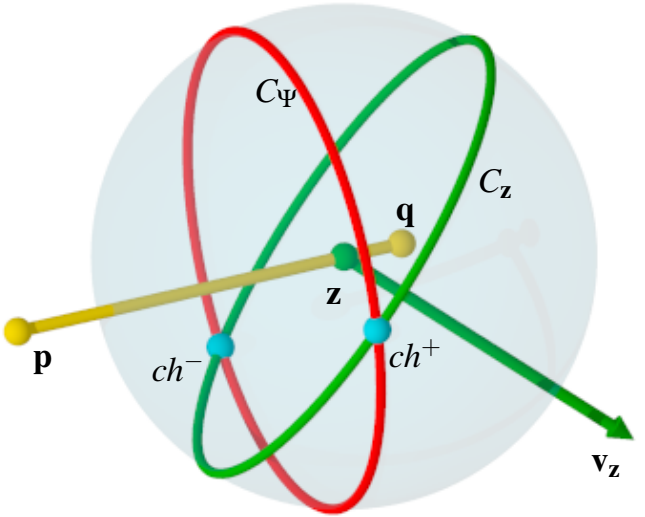

Figure 5: Construction of the characteristic. Let $\mathbf{z}$ be a given point, $\mathbf{z} \in \mathbf{p q}, \mathbf{v}_{\mathbf{z}}$ be its instantaneous velocity vector, and $C_{\mathbf{z}}$ be a great circle centered at $\mathbf{z}$ which plane is perpendicular to $\mathbf{v}_{\mathbf{z}}$. The sphere (transparent) centered at $\mathbf{z}$ touches the surface of revolution $\Psi$ along a circle $C_{\Psi}$. Then the points of the characteristic are (in general) two intersection points (blue) $c h^{ \pm}=C_{\Psi} \cap C_{\mathbf{z}}$.

degenerated motions in our considerations as we are interested in flank-milling applications and we seek motions similar to the one shown in Fig. 4

To compute points of the envelope, one can consider the motion of $\Psi$ as a two-parameter family of spheres, one parameter controls the position of the sphere in the axial direction $(s)$, and the second parameter corresponds to time $(t)$. Let $\mathbf{z}=R(t, s)$ be a point on the ruled surface and let $S(s, t)$ be the corresponding sphere (the sphere radius is constant in $t$ but varies in $s$ ).

Let $C_{\Psi}$ be a contact circle between the sphere $S(s, t)$ and $\Psi(t)$ and let $C_{\mathbf{z}}$ be a great circle centered at $\mathbf{z}$ perpendicular to the velocity vector $\mathbf{v}_{\mathbf{z}}$, see Fig. 5. Then the points of the characteristic (and for variable $t$ points of the envelope) are the intersection points of $C_{\Psi}$ and $C_{\mathbf{z}}$.

\subsection{Tangential contact between two surfaces}

Let $\Phi$ be the input (design) surface and $\Psi$ be the (yet unknown) surface of revolution. For flank milling, $\Psi$ and $\Phi$ need to have a tangential contact throughout the whole motion. Let $\mathbf{p}_{i}$ be a sample point on $\Psi$ 's axis and let $\mathbf{p}_{i}^{\perp}$ be its closest point (footpoint) on $\Phi$, see Fig. 6 Representing $\Psi$ as a one-parameter family of spheres, this tangential contact means that every sphere touches $\Phi$, i.e., the surface normal of $\Phi$ at $\mathbf{p}_{i}^{\perp}$ coincides with $\mathbf{p}_{i} \mathbf{p}_{i}^{\perp}$. Given the axis $\mathbf{l}$, the locus of footpoints $\mathbf{p}_{i}^{\perp}$ defines a characteristic (and consequently one-parameter family of spheres). However, such an arrangement need not be tangentially movable along $\Phi$. The tangential movability requirement can be formulated as

$$
F(\mathbf{x})=\frac{1}{n} \sum_{i=1}^{n}\left\langle\mathbf{v}_{i}, \frac{\mathbf{p}_{i}-\mathbf{p}_{i}^{\perp}}{\left\|\mathbf{p}_{i}-\mathbf{p}_{i}^{\perp}\right\|}\right\rangle^{2}=\mathbf{x A} \mathbf{x}^{\mathrm{T}} \rightarrow \min ,
$$

with the constraint

$$
\mathbf{v}_{\mathbf{m}} \mathbf{v}_{\mathbf{m}}^{\mathrm{T}}=1
$$

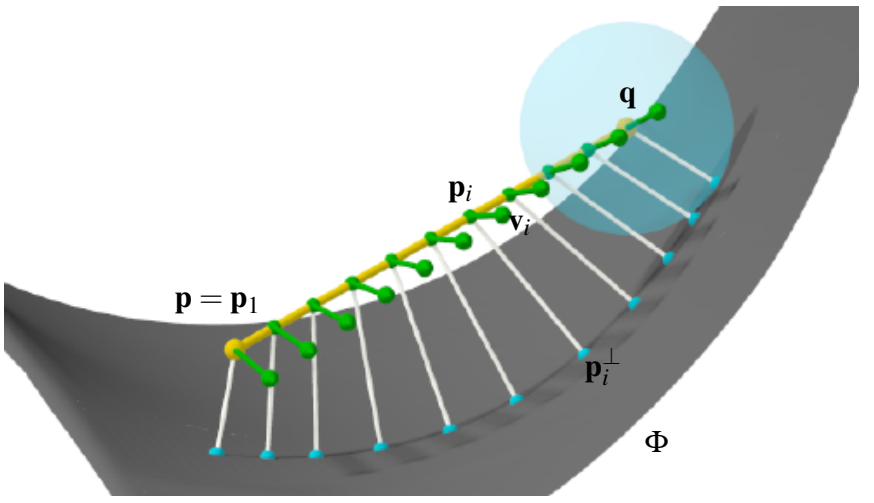

Figure 6: Tangential movability. For flank milling, the milling tool has to move tangentially along $\Phi$. The tool is conceptualized as a one-parameter family of spheres centered along pq (sphere centered at $\mathbf{q}$ shown in transparent) and an instantaneous motion (green) should move the spheres tangentially along $\Phi$. This objective is interpreted as the orthogonality between the velocity vector $\mathbf{v}_{i}$ at $\mathbf{p}_{i}, \mathbf{p}_{i} \in \mathbf{p q}$, and the line $\mathbf{p}_{i} \mathbf{p}_{i}^{\perp}, \mathbf{p}_{i}^{\perp}$ being the footpoint of $\mathbf{p}_{i}$. The orthogonality is achieved in the least square sense and the "as orthogonal as possible" vector field is computed from Eq. 4.

where $\mathbf{x}$ is the unknown instantaneous motion of $\mathbf{l}, n$ is the number of sampled points $\mathbf{p}_{i}$ on $\mathbf{l}, \mathbf{p}_{i}^{\perp}$ are their orthogonal projections onto $\Phi, \mathbf{v}_{i}$ are the velocity vectors associated to $\mathbf{p}_{i}$, and $\mathbf{v}_{\mathbf{m}}$ is the velocity at the midpoint $\mathbf{m}$ of $\mathbf{l}$, see Fig. 6

(a)
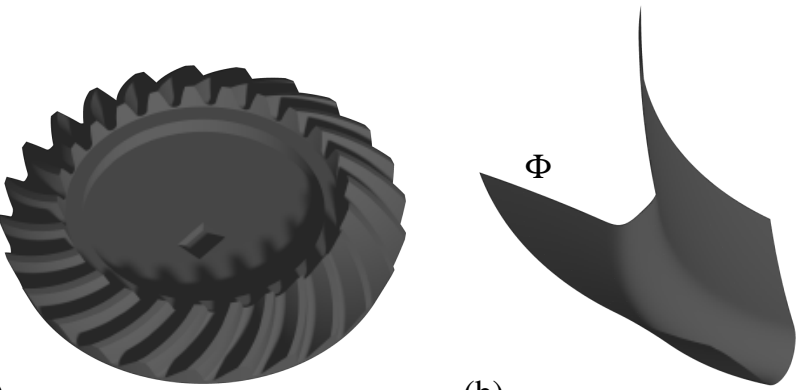

(b)

Figure 7: Test geometry. (a) A gear with curved teeth. (b) One "valley" between two teeth that is formed by a doubly curved free-form surface $\Phi$.

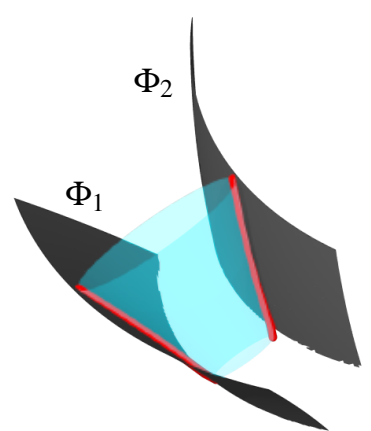

(a)

Figure 8: "Double-flank" milling. (a) We aim at designing a tool (transparent) and its motion such that the two branches of the characteristic (red) approximate well the sides of the valley, surfaces $\Phi_{1}$ and $\Phi_{2}$. (b) The motion of the tool should remove as much material as possible (green volume), yet be penetrationfree with $\Phi_{1}$ and $\Phi_{2}$. 


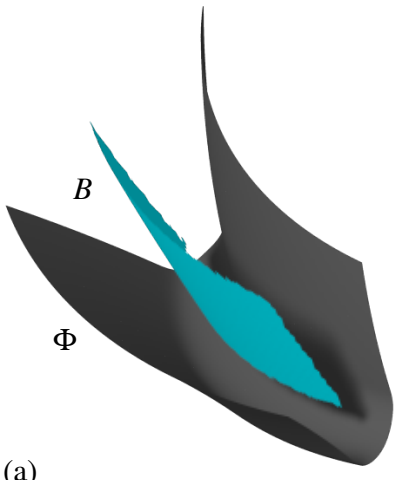

(a)

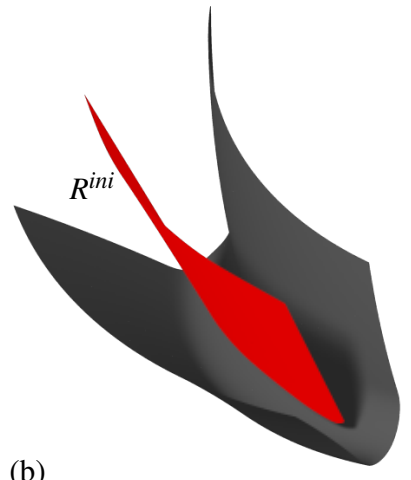

(b)

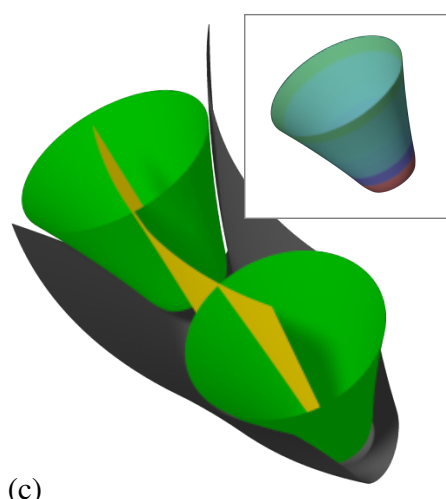

(c)

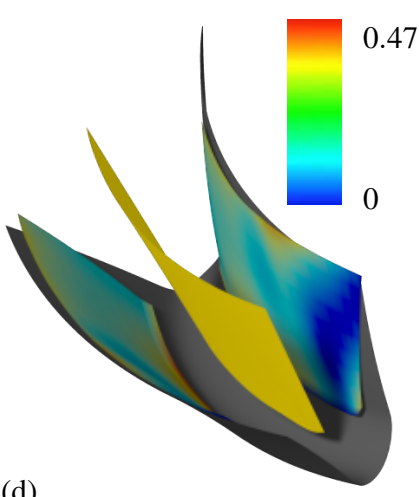

(d)

Figure 9: Algorithm pipeline. (a) The reference surface valley $\Phi$ determines its self-bisector $B$ (blue), that is approximated by the best ruled surface fit $R^{\text {ini }}$ (b). (c) The initial ruled surface (trajectory of the tool) and the shape of the tool both undergo optimization that maximizes material removal induced by the swept volume of the tool (green). The optimized custom-shaped tool (framed) is color-coded by the Gaussian curvature ranging between $K_{\text {min }}=-0.0055$ (blue) and $K_{\max }=0.0824$ (red, the tool-tip). (d) The two envelopes, color-coded by the distance error from $\Phi$, are as close as possible to $\Phi$ and, by construction, guaranteed to be penetration-free with $\Phi$.

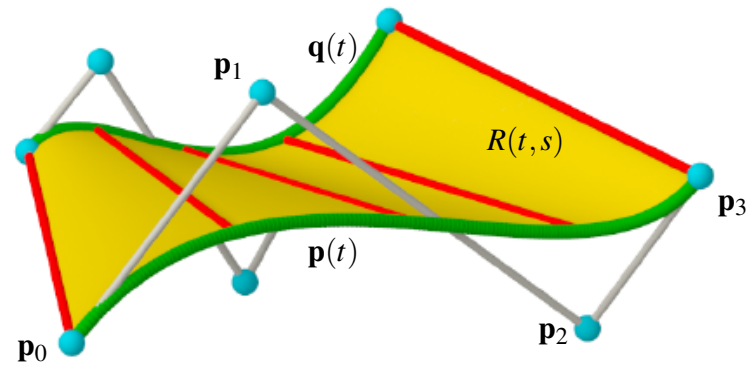

Figure 10: Ruled surface $R(t, s)$ is realized as a tensor product B-spline patch, linear in the $s$-direction (rulings) and cubical in the $t$-direction (time). Here only one polynomial segment in Bézier representation is shown. Two boundary curves $\mathbf{p}(t)$ and $\mathbf{q}(t)$ (green) are determined by their control points (blue), which are the unknowns in our optimization setup.

The number of degrees of freedom to move a line in $3 \mathrm{D}$ is five, therefore $\mathbf{x} \in \mathbb{R}^{5}$. Note that if $\Phi$ is an exact envelope, the solution $\mathbf{x}^{*}$ of (4) vanishes, i.e., $F\left(\mathbf{x}^{*}\right)=0$. Since $F$ is quadratic in $\mathbf{x}$, the unique minimizer of (4) is (in the least square sense) the best motion that moves the line (and consequently the associated one parameter family of spheres) as tangentially as possible along $\Phi$.

\section{Fitting free-form surfaces by envelopes of surfaces of rev- olution with general meridians}

We aim to approximate the input free-form surface $\Phi$ by a motion of a custom-shaped milling tool $\Psi$. In particular, in our machining application we are interested in geometries where one can speak about "valleys" as is the case of the space between two teeth of a spiral bevel gear, see Fig. 7. Our objective is to define a shape of the tool for the semi-finishing part of machining such that the material removal will be as efficient as possible, yet will be penetration-free. We aim to remove material on both sides of the valley, therefore we call this methodology as double-flank milling, see Fig. 8. We now describe the particular parts of our method in more detail, Fig. 9 shows the graphic overview of our algorithm.

\subsection{Computation of a self-bisector}

To initialize the motion of the milling tool, we first compute the self-bisector of $\Phi[34]$. As the tool can be conceptualized as a one parameter family of spheres that should ideally touch $\Phi$ on two sides, the self-bisector $B$ is a locus of all such centers of spheres.

We first pre-process the geometry by splitting the input valley $\Phi$ into the right and left side (defined by trimming off the bottom part of the valley), yielding two surfaces $\Phi_{1}$ and $\Phi_{2}$, see Fig. \& To compute $B$, we use a variant of the marching cubes algorithm as follows. We define a trivariate function $F(\mathbf{z}), \mathbf{z} \in \mathbb{R}^{3}$ as

$$
F(\mathbf{z})=\operatorname{dist}\left(\mathbf{z}, \Phi_{1}\right)-\operatorname{dist}\left(\mathbf{z}, \Phi_{2}\right),
$$

where $\mathbf{z} \in \mathbb{R}^{3}$ is the desired center of the sphere and dist is the point-surface minimal distance. We seek the iso-surface $F(\mathbf{z})=$ 0 that defines our bisector $B$. This procedure returns a dense set of $3 \mathrm{D}$ point that lie on the bisector surface. B-spline fitting is sequentially used to get a smooth bisector, see Fig.9.a).

\subsection{Initial ruled surface}

For a general valley, however, the bisector $B$ is not a ruled surface. To initialize the motion of the tool axis, we need to find a good approximation of $B$ by a ruled surface $R^{\text {ini }}$, see Fig. 9 (b). We define a ruled surface as

$$
R(t, s)=(1-s) \mathbf{p}(t)+s \mathbf{q}(t), \quad[t, s] \in[0,1] \times[0,1],
$$

where $\mathbf{p}(t)$ and $\mathbf{q}(t)$ are the two boundary (aka rail) 3D curves. In our implementation, a ruled surface is realized as a B-spline

\footnotetext{
${ }^{1}$ Observe that the geometry of the very bottom part of $\Phi$ (the bottom of the valley) prevents to use flank milling methodology. In real milling, this part is typically milled by 5 -axis ball-end approach.
} 
patch of bi-degree $(1,3)$, that is linear along the rulings $(s$ direction) and cubical in the $t$-direction, see Fig. 10 .

To compute the initial approximation of $B$, we follow the approach of [6] and use the discrete rulings as uniform samples in the $t$-direction. Our (finite) ruled surface corresponds to a motion of the tool axis and therefore the Euclidean distance of the finite ruling is preserved, i.e., $\|\mathbf{p}(t) \mathbf{q}(t)\|=L, \forall t \in[0,1]$.

(a)

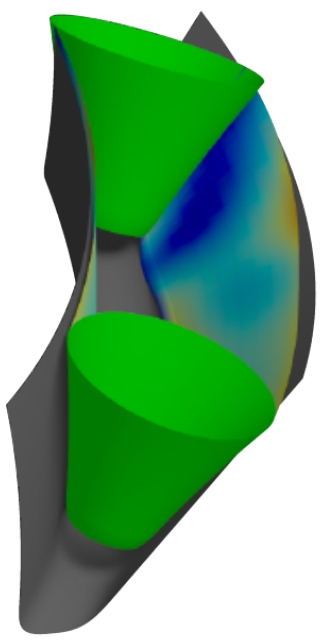

(b)

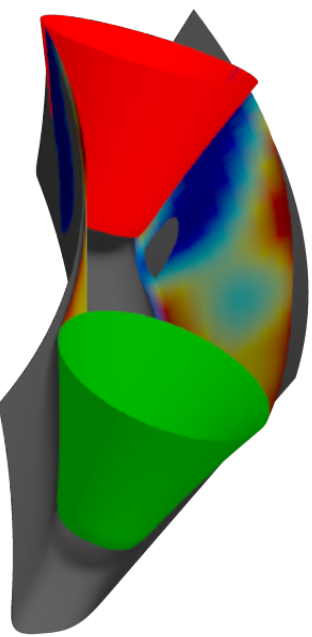

Figure 11: Controlled overcutting. (a) Penetration-free tool and its envelope. (b) Increasing the penetration-free estimate $[8$ by $2 \%$ results in more exact envelope, however, the overcutting occurs in the neighborhood of the tool at one end (red).

\subsection{Tool and motion optimization}

Once an initial ruled surface $R^{i n i}$ is computed, we have, for each value of $s, s \in[0,1]$, a set of scalar values that correspond to the distance $\operatorname{dist}(R(t, s), \Phi)$ and averaging these values for various $t$ gives the initial radial function $d^{\star}(s)$. The envelope of this one-parameter family of spheres defines the initial tool $\Psi^{i n i}$, see Fig. 2, however, the motion of $\Psi^{i n i}$ in general penetrates $\Phi$.

The ruled surface $R$ is uniformly sampled both in $t$ and $s$ parametric directions to obtain $\mathbf{r}_{i j}:=R\left(t_{i}, s_{j}\right), i=1, \ldots, m$, $j=1, \ldots, n$. Our aim is to define a tool and its motion that removes as much material as possible, yet at the same time is penetration-free with $\Phi$.

In our discrete approach, for each $s$-parameter value (fixed $j$ ), we obtain a set of discrete values $d_{i j}$ which are the distances from $\Phi$ as the fixed point of the axis moves in time. In order to obtain a motion of $\Psi$ that is penetration-free, we define

$$
\underline{d}_{j}=\min _{i=1, \ldots, m} d_{i j}
$$

$\underline{d}_{j}$ being the penetration-free radii ${ }^{2}$ These radii are the lower bounds of the point-surface distance for each $j$, i.e., distances

\footnotetext{
${ }^{2}$ The penetration-free radius depends on the sampling density. In our experiments $m=100$ turned out to be sufficiently large to return stable values for the valley shown in Fig. 7
}

that define (discrete) radial function that corresponds to a penetrationfree tool, see Fig. 11 We further define the penetration-free error as

$$
\varepsilon_{j}=d_{j}^{\star}-\underline{d}_{j}
$$

where $d_{j}^{\star}$ are the samples of the initial radial function $d^{\star}$. We denote by $\mathbf{d}$ a vector of unknown distances $\mathbf{d}:=\left(d_{1}, \ldots, d_{n}\right)$ and optimize both, the ruled surface $R$ and $\mathbf{d}$.

Remark 3. While our goal is to remove as much material as possible, our primary objective is to prevent overcutting and therefore we look for a tool and its motion that is penetrationfree. The definition of the penetration-free radii (8) is conservative and therefore there is a small gap between the tool and the design surface. Increasing the penetration-free conservative bound (8) by only $2 \%$ increases the tool size (and consequently the material removal), however, it causes the tool to penetrate the surface, see Fig. 11 and 12 Therefore we consider our conservative bound (8) a very good approximation of the optimal radial function.

At every time instant $t$, the tool is required to be as close as possible to $\Phi$, yet penetration-free. The approximation objective is formulated as a minimization problem

$$
F_{\text {prox }}(\mathbf{p}, \mathbf{q}, \mathbf{d})=\frac{1}{m n} \sum_{j=1}^{n} \sum_{i=1}^{m}\left(\operatorname{dist}\left(\mathbf{r}_{i j}, \Phi\right)-d_{j}-\varepsilon_{j}\right)^{2} \rightarrow \min
$$

subject to the rigidity constraints

$$
F_{\text {rigid }}(\mathbf{p}, \mathbf{q})=\left\langle\mathbf{p}\left(t_{i}\right)-\mathbf{q}\left(t_{i}\right), \mathbf{p}\left(t_{i}\right)-\mathbf{q}\left(t_{i}\right)\right\rangle-L^{2}=0,
$$

where $\operatorname{dist}($,$) is a point-surface distance and L$ is the length of $\mathbf{l}$. The unknowns in the minimization are the control points of the two B-spline curves $\mathbf{p}(t)$ and $\mathbf{q}(t)$, and the vector of sphere radii d, see Fig. 10. If not stated differently, $m=100$ and $n=30$ are used in our computations.

Further denote by $\mathbf{r}_{i j}^{\perp}$ the footpoints of $\mathbf{r}_{i j}$ on $\Phi$, and $\mathbf{n}_{i j}$ the unit normals at $\mathbf{r}_{i j}^{\perp}$ oriented towards $\mathbf{r}_{i j}$. We define the pointsurface proximity constraints as

$$
\begin{aligned}
& F_{\text {point }}(\mathbf{p}, \mathbf{q}, \mathbf{d})=\frac{1}{m n} \sum_{j=1}^{n} \sum_{i=1}^{m}\left\|\mathbf{r}_{i j}-\left(\mathbf{r}_{i j}^{\perp}+\left(d_{j}+\varepsilon_{j}\right) \mathbf{n}_{i j}\right)\right\|^{2}, \\
& F_{\text {plane }}(\mathbf{p}, \mathbf{q}, \mathbf{d})=\frac{1}{m n} \sum_{j=1}^{n} \sum_{i=1}^{m}\left(\left\langle\mathbf{r}_{i j}-\left(\mathbf{r}_{i j}^{\perp}+\varepsilon_{j} \mathbf{n}_{i j}\right), \mathbf{n}_{i j}\right\rangle-d_{j}\right)^{2},
\end{aligned}
$$

which correspond to a point-point and point-plane distance constraints, respectively. Note that the discrete samples $d_{j}, j=$ $1, \ldots, n$ of the radial distance function are the unknowns, while the penetration-free errors $\varepsilon_{j}, j=1, \ldots, n$ are constant. Formulation of (12) expresses our wish that the radial distance function attains maximal values which are still under the penetration error expressed by $\varepsilon_{j}$ s.

To achieve a fair motion, fairness of the two boundary curves is expressed by

$$
\begin{aligned}
F_{\text {fair }}(\mathbf{p}, \mathbf{q}) & =\frac{1}{m} \sum_{i=2}^{m-1}\left(\mathbf{p}\left(t_{i-1}\right)-2 \mathbf{p}\left(t_{i}\right)+\mathbf{p}\left(t_{i+1}\right)\right)^{2} \\
& +\frac{1}{m} \sum_{i=2}^{m-1}\left(\mathbf{q}\left(t_{i-1}\right)-2 \mathbf{q}\left(t_{i}\right)+\mathbf{q}\left(t_{i+1}\right)\right)^{2} .
\end{aligned}
$$



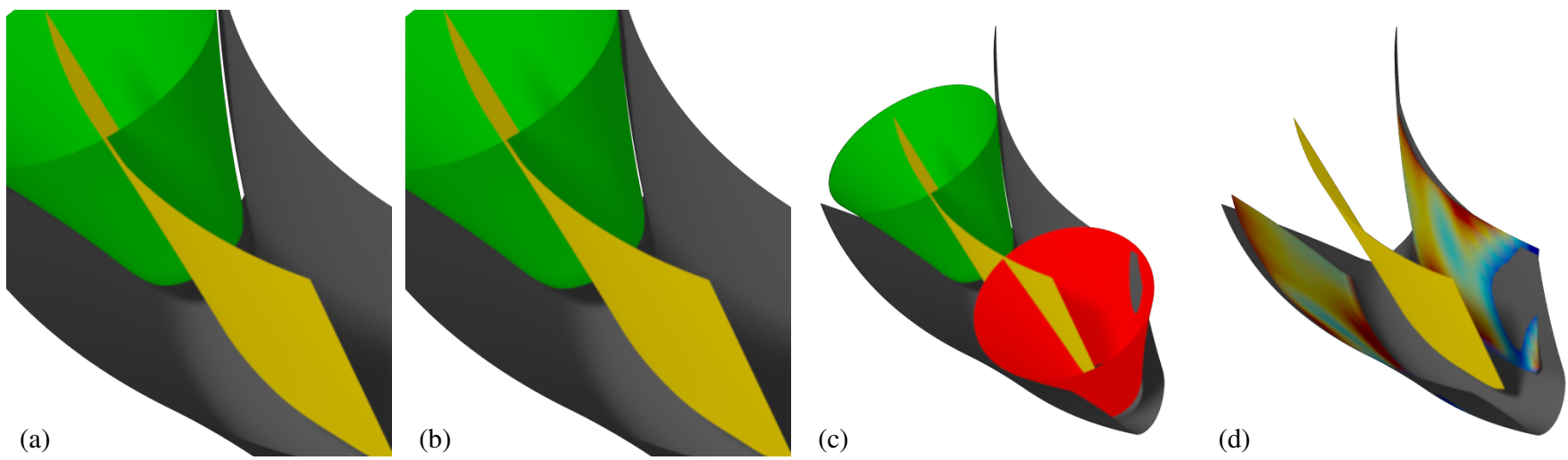

Figure 12: Penetration-free estimate. (a) A small gap between the tool and the reference surface (a zoom-in of Fig. 9.c)). (b) Increasing our conservative penetrationfree radius estimate 8 by only $2 \%$ increases the tool size. (c) However, this causes the tool-vs-surface interference (red) at some other location. (d) The two envelope surfaces; one pentrating $\Phi$ that corresponds to overcutting.

Moreover, to make the milling path efficient, the motion of the tool is required to move in a direction that is as orthogonal to the axis as possible which is expressed as

$$
\begin{aligned}
F_{\text {ortho }}(\mathbf{p}, \mathbf{q}) & =\frac{1}{m} \sum_{i=1}^{m-1}\left\langle\frac{\mathbf{p}\left(t_{i+1}\right)-\mathbf{p}\left(t_{i}\right)}{\left\|\mathbf{p}\left(t_{i+1}\right)-\mathbf{p}\left(t_{i}\right)\right\|}, \mathbf{e}_{i}\right\rangle \\
& +\frac{1}{m} \sum_{i=1}^{m-1}\left\langle\frac{\mathbf{q}\left(t_{i+1}\right)-\mathbf{q}\left(t_{i}\right)}{\left\|\mathbf{q}\left(t_{i+1}\right)-\mathbf{q}\left(t_{i}\right)\right\|}, \mathbf{e}_{i}\right\rangle .
\end{aligned}
$$

where $\mathbf{e}_{i}$ is the unit directional vector of pq.

The final objective function reads as

$$
\begin{aligned}
F_{\text {motion }}(\mathbf{p}, \mathbf{q}, \mathbf{d})=\mu_{1} F_{\text {plane }}(\mathbf{p}, \mathbf{q}, \mathbf{d}) & +\mu_{2} F_{\text {fair }}(\mathbf{p}, \mathbf{q}) \\
& +\mu_{3} F_{\text {point }}(\mathbf{p}, \mathbf{q}, \mathbf{d})+\mu_{4} F_{\text {rigid }}(\mathbf{p}, \mathbf{q}) \\
& +\mu_{5} F_{\text {ortho }}(\mathbf{p}, \mathbf{q})
\end{aligned}
$$

where the axis tool rigidity (11) is added as a soft-constraint. We solve the optimization problem using the Gauss-Newton and, if not stated differently in Section 5, the default values $\mu_{1}=1, \mu_{2}=\mu_{4}=\mu_{5}=0.1$, and $\mu_{3}=0.001$ are used.

\section{Results and validation}

In order to validate the results obtained by our algorithm, commercial CAD/CAM/CAE software (NX from Siemens) was selected. This type of software is commonly used for manufacturing processes to generate tool-paths for conventional and non-conventional processes, such as milling, turning or grinding. Additionally, this software contains a module for running a dimensional accuracy comparison between the desired design and the final one obtained using a given tool-path. In this line, Fig. 13 and the attached video shows the comparison performed introducing the tool-path generated using our algorithm with the final expected gear design. Fig. 13a shows the tool-path implementation from the algorithm to the CAM software, Fig. 13 p and c show the initial blank definition and the cutting estimation. It was confirmed by the NX software that only overcut (extra material) of $0.15-0.40 \mathrm{~mm}$ occurs, avoiding everywhere the undercut error that is considered critical to preserve the dimensional requirements. Hence, the simulations using the commercial software confirm the error obtained in our computations and also the claim that the tool path is penetration-free with the gear geometry.

The prototype of the custom-shaped milling tool for our specific gear geometry was 3D-printed, see Fig. 14. The SST $1200 \mathrm{em} 3 \mathrm{D}$ printer was used with $\mathrm{ABS}+$ polymer as a printing material and the resolution was set to $0.254 \mathrm{~mm}$ per layer. Geometrically, the virtual tool is hyperbolic in the upper part closer to the base, and elliptic close to the tool-tip, see Fig.9. (c) for the plot of Gaussian curvature. Due to the resolution, the feature of the elliptic tool-tip is not captured well in the 3D printed prototype, see Fig. 14

\subsection{Physical experiments and validation with prototyped tool and piece}

The computer simulations of double-flank milling were validated by conducting physical experiments in the machining center IBARMIA ZV-25/U600 model (IBARMIA INNOVATEK S.L.U., Guipuzkoa, Spain), being numerically controlled by Heidenhain iTNC530, see Fig. 15. This machining center is a five-axis machine with three linear axes, two rotary axes, and a spindle capacity of $18,000 \mathrm{rpm}$ and $18 \mathrm{KW}$.

The physical experiments were performed with the prototyped tool and piece (both 3D printed, see Fig. 14). The tool and piece were both manufactured in a 3D printer Stratasys SST 1200es (STRATASYS LDT., Minnesota, United States). The accuracy of the prints is related to the layer thickness. In SST 1200es, two different layer thickness could be applied: $0.254 \mathrm{~mm}(0.010$ inches $)$ and $0.33 \mathrm{~mm}(0.013$ inches $)$. In this experiment, $0.254 \mathrm{~mm}$ was selected for the layer thickness of the printed parts. The resolution in the XY plane is overtaken by the layer thickness resolution. 3D printer resolution is also related to the printing temperature which in our experiment was between $18-30^{\circ} \mathrm{C}$.

In order to validate the double-tangential contact, the tool flank was painted in red and after motion performance, the red paint got transferred onto the gear proving the contact with the 

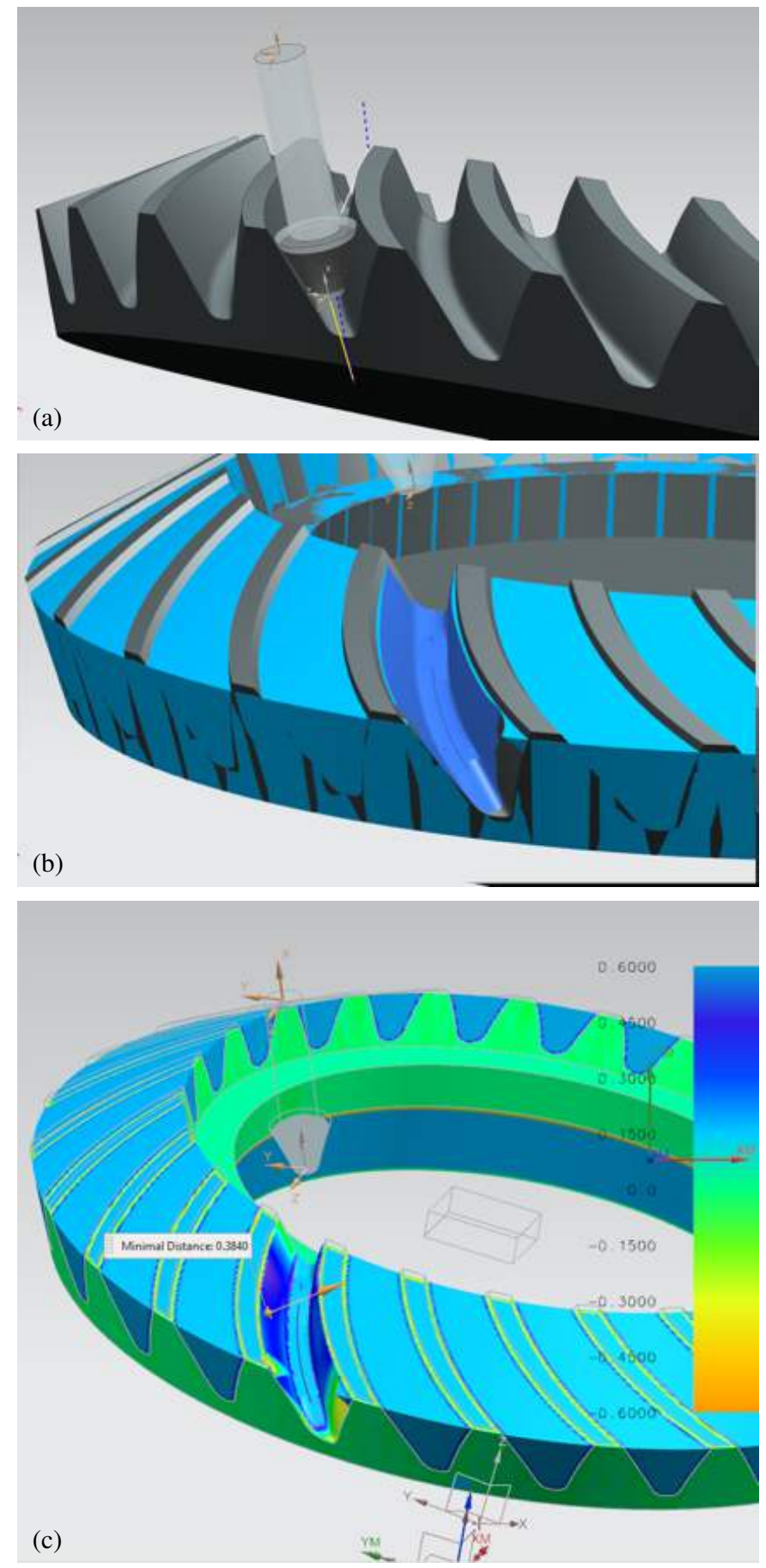

Figure 13: Verification using commercial software Siemens NX. (a) The tool is navigated along the reference gear geometry using our result shown in Fig.9. c). (b) The material to be removed (light blue) and one path of our custom-shaped tool (dark blue) are shown. (c) The path of tool is color coded by the error and is penetration-free everywehre (note that the yellow regions that correspond to overcutting are located outside our tool path).

teeth on both sides of the valley. Neither the tool nor the piece were scratched after a single-sweep experiment, validating also the very fine precision obtained in the numerical simulation stage. The toolpath shown in Fig. 11.a) was used for the physical realization.
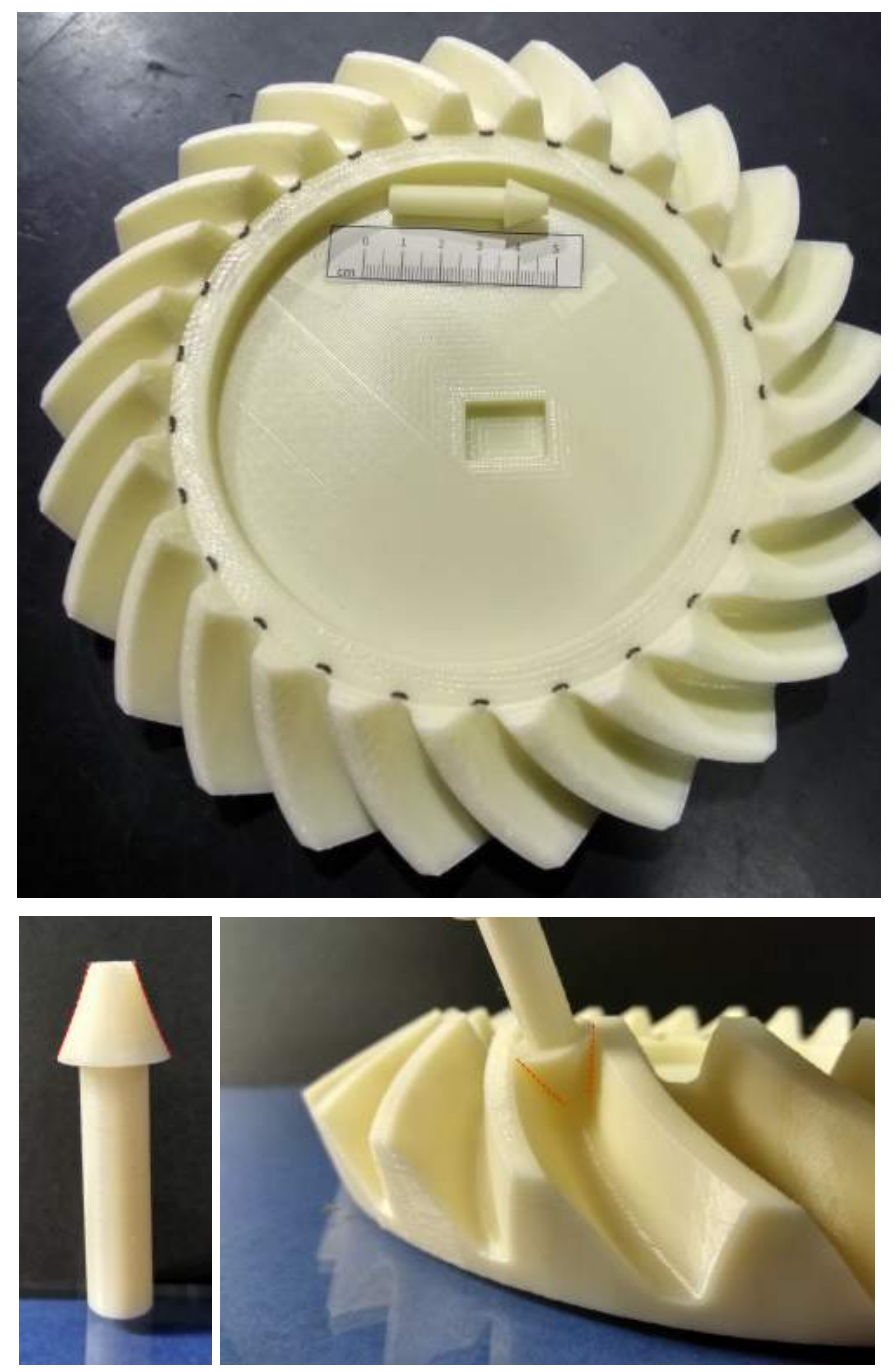

Figure 14: 3D printed prototype. Top: The tool and the gear geometry were both $3 \mathrm{D}$ printed. The ruler shows the scale of the tool, which axial length is cca $1 \mathrm{~cm} ; 4 \mathrm{~cm}$ including the cylindrical handler. Bottom left: A zoom-in of the custom-shaped tool with a curvature-varying meridian (red). Bottom right: the initial position of the tool w.r.t the gear geometry. For double-flank milling, the tool posses a tangential contact along two curves (red).

\subsection{Discussion and Limitations}

Our results show that the introduced double-flank milling methodology is well-suited for gear geometry, however, one should not misinterpret our result as there is no guarantee that every (bevel) gear can be well-approximated by a double-envelope of a single sweep of a custom-shaped tool. One can construct counter examples where such an approach is barely possible. Consider, for example, a surface (valley) that arises by extruding a planar profile that is convex on one side and concave on the other. If the extrusion direction is perpendicular to the plane of the profile, the double tangential contact by a rotational tool would not be possible, assuming the machining constraint that the handle of the tool has to hold the tool from above the valley. A better mathematical classification of surfaces where the double-tangential contact is possible is one avenue for our future research. 


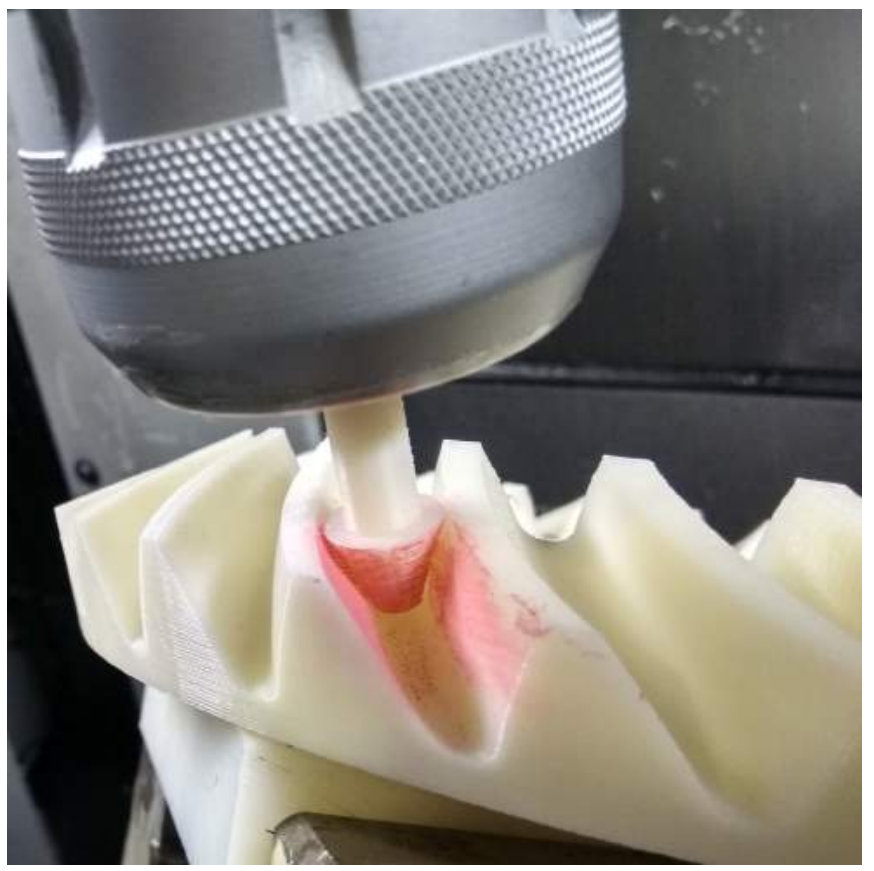

Figure 15: Physical validation. The 3D printed prototype of the milling tool was painted in red to mark the tangential contact with both sides of the teeth. Neither the tool nor the piece carried away marks of damage from the test, demonstrating double-tangential contact with a very fine precision.

Another issue that can still be improved is the initialization stage where a ruled surface is fitted to the bisector. Currently we fit the ruled surface without using the information of the distance field. Therefore a more advanced algorithm that takes into account not only the bisector surface, but also the scalar function, should improve the accuracy of the initialization.

Another limitation stems in the fact that the physical validations were performed using a 3D-printed prototype of the tool and the workpiece and therefore the quality (and inaccuracy) of the 3D printing directly affects the path-planning results. A thorough experiments using a metal variant of the tool, followed by quality inspection, are needed to fully support double-flank milling as a legitimate methodology for gear manufacturing.

\section{Conclusion and Future Work}

We have introduced a new machining methodology called double-flank milling. This methodology is aimed at narrow regions, like valleys between two curved teeth of a spiral bevel gear, where the milling tool can move in double tangential contact along the reference geometry. The tool and its 3D motion are the unknowns in our optimization-based algorithm which looks for the tool of largest possible size such that the envelope of its motion is penetration-free with the input surface. We have demonstrated our algorithm on a gear model with curved teeth and validated it using both commercial software accuracy simulation and physical realizations. A prototype of the customshaped milling tool and the piece have been $3 \mathrm{D}$ printed and used for a preliminary physical test. The physical test for tool positioning testing demonstrates, through a method analogous to the prussian blue accuracy testing method, that the tool position and movement is adequate. Our follow-up research aims at manufacturing a metal variant of the 3D-printed prototype to be used for real machining. This research will lead to further challenges such as cutting forces, tool vibrations, or surface roughness.

Acknowledgments. The first author has been partially supported by the National Key R\&D Program of China (2018YFB1702900), the National Natural Science Foundation of China (61672187), and the Shandong Provincial Key R\&D Program (2018GGX103038). The second and third authors have been supported by the Spanish Ministry of economy (MIMECOR) Implantacin de una solucin integral para la fabricacin y reparacin de componentes de turbo maquinaria (Turbo) and by DP12016-74845-R Estrategias avanzadas de definicin de fresado en piezas rotativas integrales, con aseguramiento de requisito de fiabilidad y productividad. The last author has been partially supported by the Basque Government through the BERC 2014-2017 program and by Spanish Ministry of Science, Innovation and Universities: Ramón y Cajal with reference RYC-2017-22649. The project leading to this application has received funding from the European Unions Horizon 2020 research and innovation programme under agreement No. 862025.

\section{References}

[1] Ming Luo, Dongqing Yan, Baohai Wu, and Dinghua Zhang. Barrel cutter design and toolpath planning for high-efficiency machining of freeform surface. The International Journal of Advanced Manufacturing Technology, 85(9-12):2495-2503, 2016.

[2] Jérémie Dumas, Jean Hergel, and Sylvain Lefebvre. Bridging the gap: automated steady scaffoldings for 3d printing. ACM Transactions on Graphics (TOG), 33(4):98, 2014.

[3] Xiaoting Zhang, Xinyi Le, Athina Panotopoulou, Emily Whiting, and Charlie CL Wang. Perceptual models of preference in $3 \mathrm{~d}$ printing direction. ACM Transactions on Graphics (TOG), 34(6):215, 2015.

[4] A Calleja, MA Alonso, A Fernández, I Tabernero, I Ayesta, A Lamikiz, and LN López de Lacalle. Flank milling model for tool path programming of turbine blisks and compressors. International Journal of Production Research, 53(11):3354-3369, 2015.

[5] Pengbo Bo, Michael Bartoň, Denys Plakhotnik, and Helmut Pottmann. Towards efficient 5-axis flank CNC machining of free-form surfaces via fitting envelopes of surfaces of revolution. Computer-Aided Design, 79:1$11,2016$.

[6] H. Y. Chen and H. Pottmann. Approximation by ruled surfaces. J. Comput. Appl. Math., 102:143-156, 1999.

[7] M. Peternell, H. Pottmann, and B. Ravani. On the computational geometry of ruled surfaces. Computer-Aided Design, 31:17-32, 1999.

[8] C.C.L. Wang and G. Elber. Multi-dimensional dynamic programming in ruled surface fitting. Computer-Aided Design, 51:39-49, 2014.

[9] K. Sprott and B. Ravani. Cylindrical milling of ruled surfaces. Int. J. Adv. Manuf. Technol., 38:649-656, 2008.

[10] Cornelia Menzel, Sanjeev Bedi, and Stephen Mann. Triple tangent flank milling of ruled surfaces. Computer-Aided Design, 36(3):289-296, 2004.

[11] CH Chu, WN Huang, and YY Hsu. Machining accuracy improvement in five-axis flank milling of ruled surfaces. International Journal of Machine Tools and Manufacture, 48(7):914-921, 2008.

[12] Ke Xu, Jiarui Wang, Chih-Hsing Chu, and Kai Tang. Cutting force and machine kinematics constrained cutter location planning for five-axis flank milling of ruled surfaces. Journal of Computational Design and Engineering, 2017, in press.

[13] C. Li, S. Bedi, and S. Mann. Flank milling of a ruled surface with conical tools - an optimization approach. Int. J. Adv. Manuf. Technol., 29:1115i1124,2006 
[14] J. Redonnet, W. Rubio, and G. Dessein. Side milling of ruled surfaces; optimum positioning of the milling cutter and calculation of interference. The International Journal of Advanced Manufacturing Technology, 14(7):459-465, 1998.

[15] H. Gong, C. Li-Xin, and L. Jian. Improved positioning of cylindrical cutter for flank milling ruled surfaces. Computer-Aided Design, 37:12051213, 2005.

[16] Chih-Hsing Chu, Charlie CL Wang, and Chi-Rung Tsai. Computer aided geometric design of strip using developable bézier patches. Computers in Industry, 59(6):601-611, 2008.

[17] G. Elber and R. Fish. 5-axis freeform surface milling using piecewise rule surface approximation. ASME Journal of Manufacturing Science and Engineering, 119(3):383-387, 1997.

[18] Pengbo Bo, Michael Bartoň, and Helmut Pottmann. Automatic fitting of conical envelopes to free-form surfaces for flank CNC machining. Computer-Aided Design, 91:84-94, 2017.

[19] Jinesh Machchhar, Denys Plakhotnik, and Gershon Elber. Precise algebraic-based swept volumes for arbitrary free-form shaped tools towards multi-axis cnc machining verification. Computer-Aided Design, 90:48-58, 2017.

[20] Ben Ezair and Gershon Elber. Automatic generation of globally assured collision free orientations for 5-axis ball-end tool-paths. Computer-Aided Design, 102:171-181, 2018.

[21] Amaia Calleja, Pengbo Bo, Haizea González, Michael Bartoň, and Luis Norberto López de Lacalle. Highly accurate 5-axis flank CNC machining with conical tools. The International Journal of Advanced Manufacturing Technology, pages 1-11, 2018.

[22] C. Li, S. Bedi, and S. Mann. Flank millable surface design with conical and barrel tools. Computer-Aided Design and Applications, 5:461-470, 2008.

[23] Gorka Urbikain, Egoitz Artetxe, and Luis Norberto López de Lacalle. Numerical simulation of milling forces with barrel-shaped tools considering runout and tool inclination angles. Applied Mathematical Modelling, 47:619-636, 2017.

[24] Zi Zhou, Yang Zhang, and Kai Tang. Sweep scan path planning for efficient freeform surface inspection on five-axis $\mathrm{cmm}$. Computer-Aided Design, 77:1-17, 2016.

[25] Liang Yu, Lei Zhong, and Yuhan Wang. Optimizing tool size and tool path of five-axis flank milling with bounded constraints via normal mapping. Advances in Mechanical Engineering, 9(10):1687814017724704, 2017.

[26] H. Gong and N. Wang. Optimize tool paths of flank milling with generic cutters based on approximation using the tool envelope surface. Computer-Aided Design, 41(12):981-989, 2009.

[27] L. Zhu, G. Zheng, H. Ding, and Y. Xiong. Global optimization of tool path for five-axis flank milling with a conical cutter. Computer-Aided Design, 42(10):903-910, 2010.

[28] Gang Zheng, Limin Zhu, and Qingzhen Bi. Cutter size optimisation and interference-free tool path generation for five-axis flank milling of centrifugal impellers. International Journal of Production Research, 50(23):6667-6678, 2012.

[29] L. Zhu, H. Ding, and Y. Xiong. Simultaneous optimization of tool path and shape for five-axis flank milling. Comput. Aided Des., 44:1229-1234, 2012.

[30] Liang Yu, Lei Zhong, and YuHan Wang. Shape optimization of generic rotary tool for five-axis flank milling. The International Journal of Advanced Manufacturing Technology, 93(5-8):2921-2931, 2017.

[31] Pengbo Bo and Michael Bartoň. On initialization of milling paths for flank CNC machining of free-form surfaces with curvature-varying milling tools. Computer Aided Geometric Design, in press.

[32] Helmut Pottmann and Martin Peternell. Envelopes-computational theory and applications. In Proceedings of Spring Conference on Computer Graphics, pages 3-23, 2000.

[33] H. Pottmann and J. Wallner. Computational Line Geometry. Springer, 2001.

[34] Martin Peternell. Geometric properties of bisector surfaces. Graphical Models, 62(3):202-236, 2000. 